Article

\title{
Copper-Catalyzed Dimerization/Cyclization of Itaconates
}

\author{
Zhiqiang Li, Ruirui Li, Lan Jiang and Zhengning Li * \\ College of Environmental and Chemical Engineering, Dalian University, Dalian 116622, China; \\ E-Mails: zqli501@163.com (Z.L.); 1rrnice@126.com (R.L.); jianglan@dlu.edu.cn (L.J.) \\ * Authors to whom correspondence should be addressed; E-Mail: znli@dl.cn; \\ Tel.: +86-411-8740-3576; Fax: +86-411-8740-2449.
}

Academic Editor: Derek J. McPhee

Received: 7 June 2015 / Accepted: 5 August 2015 / Published: 17 August 2015

\begin{abstract}
A copper-catalyzed domino reaction between itaconate esters and diethyl zinc (or silane) is developed, affording itaconate dimerization products, multi-ester-substituted cyclopentanones, in moderate to high yields.
\end{abstract}

Keywords: copper; catalysis; domino reaction; itaconate; dimerization; cyclopentanone

\section{Introduction}

The metal-catalyzed conjugate addition of organic zinc reagents, which are mild alkylating reagents, to $\alpha, \beta$-unsaturated carbonyls and esters, has been used extensively for $\mathrm{C}-\mathrm{C}$ bond formation in organic synthesis [1-7]. The enolate intermediate formed in this process or by reductive addition can further react with an electrophile under the same reaction conditions, leading to a domino mode of reaction, which is highly desirable in organic synthesis. Applications of the conjugate reduction-derivated enolates have been reported in Negishi coupling carboannulation [8], aldolization [9,10], the Mannich reaction [11-13], and Ireland rearrangement [14].

Recently, we reported a copper-promoted conjugate reductive-aldol/lactonization domino reaction of dimethyl itaconate with a silane and carbonyls [15], and reductive Mannich reaction/lactamization of dimethyl itaconate with imines [16]. As a replacement of a silane reductant, we employed diethyl zinc as a nucleophile. In this course, a reaction of diethyl zinc with dimethyl itaconate (1a) was performed, yielding 2,4-bis(methoxycarbonyl)-2-(2-methoxy-2-oxoethyl)-4-propylcyclopentanone (2a), a multi-estersubstituted cyclopentanone. Of special interest is the richness of functional groups in $\mathbf{2 a}$. Both the ketone and ester functional groups in 2a [17] and in other cyclopentanones bearing $\mathrm{CO}_{2} \mathrm{R} / \mathrm{CH}_{2} \mathrm{CO}_{2} \mathrm{R}$ 
groups [18-21] can be converted to other functional groups, making 2a and analogues valuable in organic synthesis. A literature survey indicated that there are only a few reports concerning the synthesis of this kind of molecules. A $21 \%$ and $26 \%$ yield of $\mathbf{2 a}$ and 2,4-bis(methoxycarbonyl)-2-(2-methoxy-2-oxoethyl)4-pentylcyclopentanone were obtained via a copper-catalyzed reaction of 1a with ethylaluminum dichloride [17] and $n$-butyl magnesium bromide [22], respectively. Using [Rh(COD)Cl] 2 as a catalyst, a $10 \%$ yield of substituted cyclopentanone was produced, along with $60 \%$ yield of the conjugate product benzyl succinate, via reaction of itaconate with phenylzinc chloride [1]. Given to the existence of two tertiary carbons in the product 2a, the low yields reported in literature, and low price of copper catalysts, an efficient and concise synthesis of 2a and alike compounds using copper catalysts offers very attractive prospectives. Herein, we wish to report that high yields could be achieved via double conjugate addition/cyclization domino reaction (Scheme 1) of itaconates with diethyl zinc (Scheme 2).

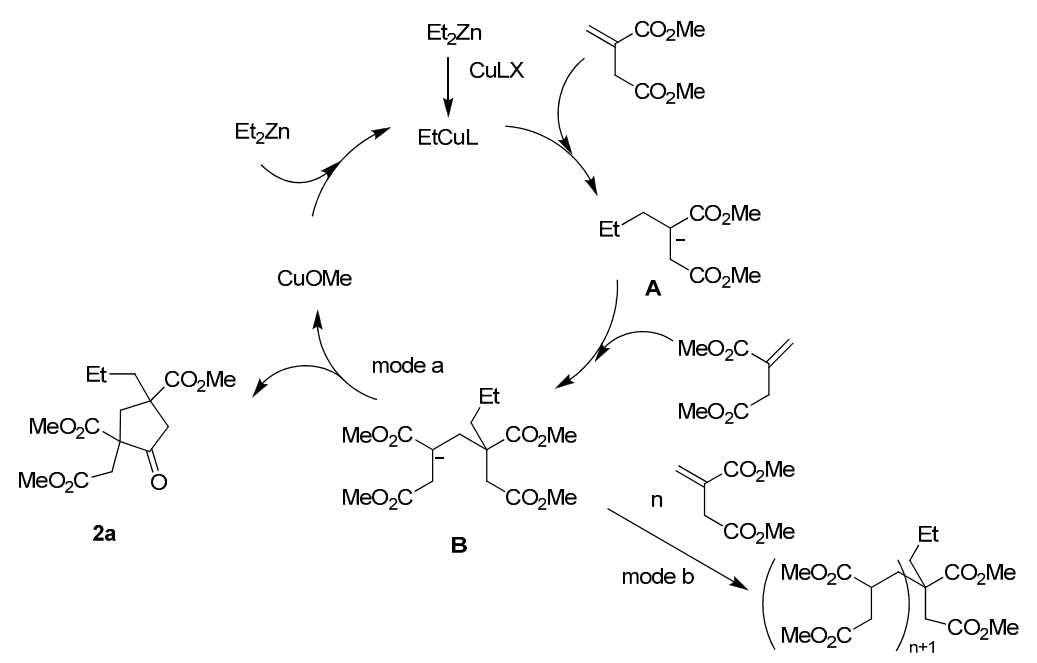

Scheme 1. Reaction pathways for formation of $\mathbf{2 a .}$

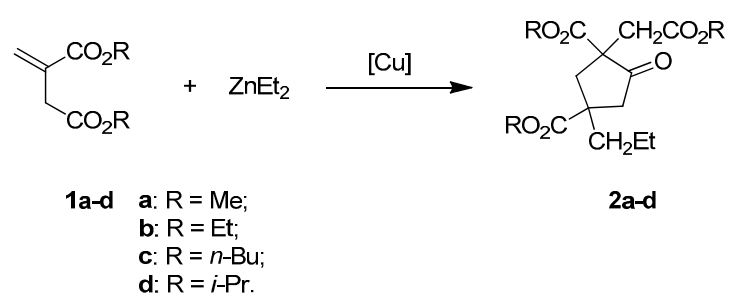

Scheme 2. Domino reactions between itaconate esters and diethyl zinc.

\section{Results and Discussion}

Reactions of diethyl zinc with 1a were performed under various conditions, under the assumption that copper catalyzed the conjugate addition of ethyl anion to itaconate, an $\alpha, \beta$-unsaturated ester, and the enolate $\mathbf{A}$ thus generated reacted with another itaconate to give enolate $\mathbf{B}$, which cyclized to afford the cyclopentanone framework and copper methoxide (Scheme 1). The results are summarized in Table 1. Using diethyl zinc as the alkylating reagent, the proposed double conjugate addition/cyclization domino reaction proceeded even in the absence of an additional catalyst, affording the cyclopentanone $2 \mathrm{a}$ in $40 \%$ yield with preference for the trans-isomer (Table 1, entry 1) [23,24]. Thus indicates the superiority of diethyl zinc over the aluminum reagent used in the literature [17]. Using $\mathrm{Cu}(\mathrm{OAc})_{2} \cdot 2 \mathrm{H}_{2} \mathrm{O}, \mathrm{CuCl}$ or $\mathrm{CuBr}$ 
as the catalyst, the yield reached $55 \%-65 \%$ with almost no diastereoselectivity (entries $2-4$ ). An increase of the yield and no change of the diastereomeric ratio were observed using $\mathrm{CuI}$ catalyst at $25{ }^{\circ} \mathrm{C}$ (entry 5). Trans-2a, assigned by its NOSEY spectrum in Supplementary Materials), was formed as the dominant diastereomer at lower temperature $\left(73 \%\right.$ at $-30{ }^{\circ} \mathrm{C}$, entry 7$)$.

Table 1. Reaction of diethyl zinc with dimethyl itaconate ${ }^{\text {a }}$.

\begin{tabular}{|c|c|c|c|c|c|c|}
\hline Entry & Cat. & Ligand & Temp $\left({ }^{\circ} \mathrm{C}\right)$ & Time (h) & Yield $^{\mathrm{b}}(\%)$ & cis/trans ${ }^{\mathrm{c}}$ \\
\hline 1 & - & - & 25 & 2.5 & 40 & $38: 62$ \\
\hline 2 & $\mathrm{Cu}(\mathrm{OAc})_{2} \cdot 2 \mathrm{H}_{2} \mathrm{O}$ & - & 25 & 4.5 & 56 & $52: 48$ \\
\hline 3 & $\mathrm{CuCl}$ & - & 25 & 2.0 & 55 & $46: 54$ \\
\hline 4 & $\mathrm{CuBr}$ & - & 25 & 2.0 & 65 & $55: 45$ \\
\hline 5 & $\mathrm{CuI}$ & - & 25 & 2.0 & 75 & $37: 63$ \\
\hline 6 & $\mathrm{CuI}$ & - & -15 & 2.0 & 61 & $30: 70$ \\
\hline 7 & $\mathrm{CuI}$ & - & -30 & 2.0 & 68 & $27: 73$ \\
\hline 8 & $\mathrm{CuFL}$ & - & 25 & 2.0 & 72 & $61: 39$ \\
\hline 9 & $\mathrm{CuFL}$ & - & 0 & 2.5 & 73 & $57: 43$ \\
\hline 10 & $\mathrm{CuFL}$ & - & -30 & 9.0 & 67 & $62: 38$ \\
\hline 11 & $\mathrm{CuFL}$ & - & -58 & 21.0 & 40 & $56: 44$ \\
\hline 12 & $\mathrm{CuFL}$ & - & -78 & 21.0 & 1 & - \\
\hline 13 & $\mathrm{CuFL}^{\mathrm{d}}$ & - & 25 & 4.0 & 53 & $55: 45$ \\
\hline 14 & $\mathrm{CuFL}^{\mathrm{e}}$ & - & 25 & 2.5 & 74 & $62: 38$ \\
\hline 15 & $\mathrm{CuFL}$ & DPPP $^{f}$ & 25 & 2.0 & 38 & $53: 47$ \\
\hline 16 & $\mathrm{CuFL}$ & DPBen $^{f}$ & 25 & 2.0 & 89 & $62: 38$ \\
\hline 17 & CuFL & DPEphos ${ }^{f}$ & 25 & 2.0 & 88 & $63: 37$ \\
\hline 18 & $\mathrm{CuFL}$ & Xantphos ${ }^{f}$ & 25 & 2.0 & $95\left(85^{g}\right)$ & $62: 38$ \\
\hline 19 & $\mathrm{CuFL}^{\mathrm{h}}$ & Xantphos & 25 & 2.5 & 98 & $64: 36$ \\
\hline
\end{tabular}

$\mathrm{CuF}\left(\mathrm{PPh}_{3}\right)_{3} \cdot 2 \mathrm{MeOH}$, with good solubility and high activity in catalyzing conjugate addition and the subsequent reaction [25-27], was employed to catalyze the reaction of 1a, and a $72 \%$ yield of 2a was obtained. Interestingly, the cis-2a isomer was the dominant one with a 61:39 cis-/trans-ratio (entry 8). Efforts to improve the diastereoselectivity using this catalyst at lower reaction temperatures (entries 9-12) were not very successful. In the meanwhile, the yields decreased. Since itaconate was consumed and almost no by-products were observed by $\mathrm{GC}$, it is reasonable to assume that the by-polymerization reaction was favored by decreasing the reaction temperature. This could be attributed to the competitive reactions of enolate $\mathbf{A}$, either cyclizing to yield $\mathbf{2}$ (mode a in Scheme 1) or undergoing conjugate addition to itaconate to yield polymer (mode $\mathrm{b}$ in Scheme 1). Anion-induced polymerization of $\alpha, \beta$-unsaturated esters, which involves conjugate addition of enolates to $\alpha, \beta$-unsaturated esters, has been reported [28]. The phenomenon that cyclization was more sensitive to temperature than conjugate addition was also observed by Shibasaki in the formation of lactams [11]. Switching the solvent to THF and dichloromethane did not show any advantages, either in terms of yield or the diastereomeric ratio (entries 13 and 14).

It should be noted that the yield is affected by the mode of addition of the reactants. Addition of diethyl zinc to a mixture of $\mathbf{1 a}$ and $\mathrm{CuF}\left(\mathrm{PPh}_{3}\right)_{3} \cdot 2 \mathrm{MeOH}$ catalyst gave much higher yield of $\mathbf{2 a}$ addition of 1a to a mixture of diethyl zinc and the catalyst, which indicates that there is not much difference 
between the rate constants of intermediate $\mathbf{A}$ and $\mathbf{B}$, and that formation of $\mathbf{B}$ is favorable at a high concentration ratio of $\mathbf{1 a}$ to diethyl zinc. Using $n$-butylzinc chloride as an alkylating reagent, the monoconjugate addition product was obtained as a dominant one. This is similar to Frost's result using phenylzinc chloride as a nucleophile under rhodium catalysis [1]. Dimethyl zinc, a less reactive reagent [29], was also tested to initialize the domino reaction, but the reaction did not proceed.

$\mathrm{CuF}\left(\mathrm{PPh}_{3}\right)_{3} \cdot 2 \mathrm{MeOH}$-diphosphorous catalysts, which have been mentioned in the literature as being advantageous in catalyzing conjugate reductions [30,31], were applied to this reaction. The introduction of 1,3-bis(diphenylphosphino)propane (dppp) decreased the yield (entry 15), while introducing 1,2-bis(diphenylphosphino)benzene (DPBen), 2,2'-bis(diphenylphosphinophenyl)ether (DPEphos), and 4,5-bis(diphenylphosphino)-9,9-dimethylxanthene (Xantphos) could increase the yield remarkably. Nevertheless, there was no great improvement of diastereomeric ratio (entries 16-18). Among the ligands screened, Xantphos gave a 95\% yield using $1.6 \mathrm{mmol}$ of dimethyl itaconate and a 98\% yield at $10 \mathrm{mmol}$ of dimethyl itaconate scale (entries 18 and 19).

Previous results indicated that using bulky alkyl $\alpha, \beta$-unsaturated esters gave higher diastereoselectivity in reductive aldol domino reactions [26]. Accordingly, we resorted to bulky alkyl groups in the itaconate. Gratifyingly, improvement of the cis-/trans-ratios was achieved using diethyl itaconate, di- $n$-butyl itaconate and di-i-propyl itaconate (Table 2, entries 2-4). However, the yields were somewhat lower.

Table 2. Reaction of diethyl zinc with dialkyl itaconate (1) a .

\begin{tabular}{ccccc}
\hline Entry & $\mathbf{1 ~ ( R )}$ & Time (h) & Yield (\%) $^{\mathbf{b}}$ & cis/trans $^{\mathbf{c}}$ \\
\hline 1 & $1 \mathrm{a}(\mathrm{Me})$ & 2 & 85 & $62: 38$ \\
2 & $1 \mathrm{~b}(\mathrm{Et})$ & 2 & 71 & $73: 27$ \\
3 & $1 \mathrm{c}(n-\mathrm{Bu})$ & 15 & 79 & $86: 14$ \\
4 & $1 \mathrm{~d}(i-\mathrm{Pr})$ & 18 & 72 & $72: 28^{\mathrm{d}}$ \\
\hline
\end{tabular}

a $11.6 \mathrm{mmol}, 1 \mathrm{Et}{ }_{2} \mathrm{Zn}: \mathrm{CuF}\left(\mathrm{PPh}_{3}\right)_{3} \cdot 2 \mathrm{MeOH}: \mathrm{Xantphos}=1.0: 0.75: 1.0 \%: 1.0 \%$ (molar ratio); ${ }^{\mathrm{b}}$ isolated yield;

${ }^{\mathrm{c}}$ determined by $\mathrm{GC}$ analysis unless noted; ${ }^{\mathrm{d}}$ determined by ${ }^{1} \mathrm{H}-\mathrm{NMR}$.

Using poly(methylhydrosiloxane) (PMHS) as a hydride source, copper-catalyzed reductive dimerization of 1a gave 2,4-bis(methoxycarbonyl)-2-(2-methoxy-2-oxoethyl)-4-methylcyclopentanone (3a) in a $41 \%$ yield (Scheme 3 ).

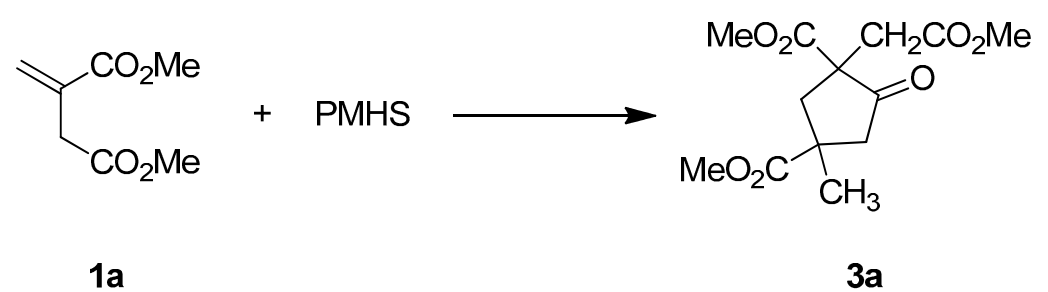

Scheme 3. A domino reaction between dimethyl itaconate and PMHS.

\section{Experimental Section}

\subsection{General Information}

All reagents were purchased from Alfa Aesar China (Shanghai, China), and the solvents were bought from Sinoreagent (Shanghai, China). GC analyses were performed on a Shimadzu GC-2010 Gas 
Chromatograph (Shimadzu Corporation, Kyoto, Japan) using an SE-54 capillary column (30 $\mathrm{m} \times$ $0.32 \mathrm{~mm} \times 0.4 \mu \mathrm{m})$. Mass spectra were recorded in electron impact mode on a HP 6890/5973 GC-MS mass spectrometer (Agilent Technologies, Santa Clara, CA, USA) at $70 \mathrm{eV}$. High_resolution mass (HRMS) analyses were performed on a Shimadzu LCMS-2020 (Shimadzu Corporation, Kyoto, Japan). Infrared spectra were recorded on a Nicolet 550 FT-IR spectrometer (Thermo Fisher Scientific Inc., Waltham, MA, USA) from $4000 \mathrm{~cm}^{-1}$ to $400 \mathrm{~cm}^{-1}$, and NMR spectra were recorded on a Bruker Adv. $500 \mathrm{NMR}$ spectrometer (Bruker Inc., Billerica, MA, USA) in $\mathrm{CDCl}_{3}$ at $500 \mathrm{MHz}$ and $125 \mathrm{MHz}$ for ${ }^{1} \mathrm{H}-\mathrm{NMR}$ and ${ }^{13} \mathrm{C}-\mathrm{NMR}$, respectively, chemical shifts are given in parts per million (ppm).

\subsection{General Procedure for the Reactions}

Under a nitrogen atmosphere and stirring, a diethyl zinc solution in hexane $(1 \mathrm{~mol} / \mathrm{L}, 1.2 \mathrm{~mL}, 1.2 \mathrm{mmol})$ was added to a dried Schlenk tube charged with Xantphos (9.3 mg, $0.016 \mathrm{mmol}),\left[\mathrm{CuF}\left(\mathrm{PPh}_{3}\right)_{3}\right] \cdot 2 \mathrm{MeOH}$ (14.9 mg, $0.016 \mathrm{mmol}), \mathbf{1 a}(0.253 \mathrm{~g}, 1.6 \mathrm{mmol})$ and toluene $(2 \mathrm{~mL})$. The mixture was stirred until the 1a was consumed, as monitored by GC analysis. A saturated ammonium chloride solution in water $(2 \mathrm{~mL})$ was added to the mixture to quench the excess diethyl zinc, and then dilute $\mathrm{HCl}$ was added to dissolve the solid. The mixture was separated by phase separation, and the aqueous solution was extracted with dichloromethane $(10 \mathrm{~mL} \times 3)$. The combined organic phase was dried and analyzed by $\mathrm{GC}$ to determine the diastereomeric ratio. Concentration of the dried organic solution and column chromatography gave 2a as a colorless oil ( 0.214 g, 85\% yield). Pure cis-2a and trans-2a could be obtained by further column chromatography on silica gel using petroleum-ethyl acetate eluent.

trans-2,4-Bis(methoxycarbonyl)-2-(2-methoxy-2-oxoethyl)-4-propylcyclopentanone (trans-2a): Colorless oil, $\mathrm{R}_{\mathrm{f}}=0.44\left(20 \%\right.$ EA in PE). ${ }^{1} \mathrm{H}-\mathrm{NMR}\left(\mathrm{CDCl}_{3}\right) \delta 3.72(\mathrm{~s}, 3 \mathrm{H}), 3.71(\mathrm{~s}, 3 \mathrm{H}), 3.67(\mathrm{~s}, 3 \mathrm{H}), 3.13(\mathrm{~d}$, $J=17.4 \mathrm{~Hz}, 1 \mathrm{H}), 3.00(\mathrm{dd}, J=17.9,1.3 \mathrm{~Hz}, 1 \mathrm{H}), 2.85(\mathrm{~d}, J=14.8 \mathrm{~Hz}, 1 \mathrm{H}), 2.66(\mathrm{dd}, J=14.8,1.4 \mathrm{~Hz}$, $1 \mathrm{H}), 2.54(\mathrm{~d}, J=17.5 \mathrm{~Hz}, 1 \mathrm{H}), 2.46(\mathrm{~d}, J=17.9 \mathrm{~Hz}, 1 \mathrm{H}), 1.93-1.84(\mathrm{~m}, 1 \mathrm{H}), 1.75-1.67(\mathrm{~m}, 1 \mathrm{H}), 1.31-1.23$ $(\mathrm{m}, 2 \mathrm{H}), 0.93(\mathrm{t}, J=7.3 \mathrm{~Hz}, 3 \mathrm{H}) .{ }^{13} \mathrm{C}-\mathrm{NMR}\left(\mathrm{CDCl}_{3}\right) \delta 209.76,176.59,171.15,170.45,58.24,53.18$, 52.40, 51.93, 47.83, 46.92, 42.03, 41.82, 38.53, 18.96, 14.28. LRMS $m / z(\%) 314\left(4,[\mathrm{M}]^{+}\right), 282(31)$, 272 (1), 255 (8), 244 (6), 223 (37), 207 (3), 195 (17), 172 (3), 156 (100), 127 (92), 113 (5), 99 (9), 85 (1), 71 (2), 55 (4). FAB-HRMS: $m / z$ calcd for $\left[\mathrm{C}_{15} \mathrm{H}_{22} \mathrm{O}_{7}+\mathrm{H}\right]^{+} 315.1438$, found 315.1440. IR $\left(\mathrm{CH}_{2} \mathrm{Cl}_{2}\right): 2956,1731,1265,733 \mathrm{~cm}^{-1}$.

cis-2,4-Bis(methoxycarbonyl)-2-(2-methoxy-2-oxoethyl)-4-propylcyclopentanone (cis-2a): Colorless oil, $\mathrm{R}_{\mathrm{f}}=0.39\left(20 \%\right.$ EA in PE). ${ }^{1} \mathrm{H}-\mathrm{NMR}\left(\mathrm{CDCl}_{3}\right) \delta 3.72(\mathrm{~s}, 3 \mathrm{H}), 3.66(\mathrm{~s}, 3 \mathrm{H}), 3.64(\mathrm{~s}, 3 \mathrm{H}), 3.31(\mathrm{dd}$, $J=19.1,1.8 \mathrm{~Hz}, 1 \mathrm{H}), 3.20(\mathrm{dd}, J=14.2,1.7 \mathrm{~Hz}, 1 \mathrm{H}), 2.96(\mathrm{~d}, J=17.6 \mathrm{~Hz}, 1 \mathrm{H}), 2.91$ (d, $J=17.6 \mathrm{~Hz}$, $1 \mathrm{H}), 2.40(\mathrm{~d}, J=19.1 \mathrm{~Hz}, 1 \mathrm{H}), 2.08(\mathrm{~d}, J=14.2 \mathrm{~Hz}, 1 \mathrm{H}), 1.89$ (ddd, $J=13.3,11.9,5.2 \mathrm{~Hz}, 1 \mathrm{H}), 1.58-1.52$ $(\mathrm{m}, 1 \mathrm{H}), 1.35-1.16(\mathrm{~m}, 2 \mathrm{H}), 0.90(\mathrm{t}, J=7.3 \mathrm{~Hz}, 3 \mathrm{H}) .{ }^{13} \mathrm{C}-\mathrm{NMR}\left(\mathrm{CDCl}_{3}\right) \delta 211.39,176.13,171.20$, $170.29,57.95,52.94,52.43,52.09,48.92,46.29,43.82,42.99,38.86,19.25,14.36$. LRMS $m / z(\%)$ $314\left(3,[\mathrm{M}]^{+}\right), 282$ (43), 272 (1), 255 (8), 244 (9), 223 (43), 207 (1), 195 (21), 172 (3), 156 (100), 127 (98), 113 (9), 99 (14), 85 (2), 71 (2), 55 (4). FAB-HRMS: $m / z$ calcd for $\left[\mathrm{C}_{15} \mathrm{H}_{22} \mathrm{O}_{7}+\mathrm{H}\right]^{+} 315.1438$, found 315.1442. IR $\left(\mathrm{CH}_{2} \mathrm{Cl}_{2}\right)$ : 2923, 1732, 1265, $737 \mathrm{~cm}^{-1}$. 
trans-2,4-Bis(ethoxycarbonyl)-2-(2-ethoxy-2-oxoethyl)-4-propylcyclopentanone (trans-2b): Colorless oil, $\mathrm{R}_{\mathrm{f}}=0.54\left(15 \%\right.$ EA in PE). ${ }^{1} \mathrm{H}-\mathrm{NMR}\left(\mathrm{CDCl}_{3}\right) \delta 4.26-4.05(\mathrm{~m}, 6 \mathrm{H}), 3.13(\mathrm{~d}, J=17.5 \mathrm{~Hz}, 1 \mathrm{H}), 2.98$ $(\mathrm{d}, J=17.0 \mathrm{~Hz}, 1 \mathrm{H}), 2.85(\mathrm{~d}, J=14.8 \mathrm{~Hz}, 1 \mathrm{H}), 2.66(\mathrm{dd}, J=14.7,0.8 \mathrm{~Hz}, 1 \mathrm{H}), 2.51(\mathrm{~d}, J=17.5 \mathrm{~Hz}$, $1 \mathrm{H}), 2.44$ (d, $J=17.9 \mathrm{~Hz}, 1 \mathrm{H}), 1.90$ (ddd, $J=13.2,11.0,5.8 \mathrm{~Hz}, 1 \mathrm{H}), 1.70$ (ddd, $J=13.4,10.8,5.9 \mathrm{~Hz}$, $1 \mathrm{H}), 1.33-1.20(\mathrm{~m}, 11 \mathrm{H}), 0.93(\mathrm{t}, J=7.3 \mathrm{~Hz}, 3 \mathrm{H}) .{ }^{13} \mathrm{C}-\mathrm{NMR}\left(\mathrm{CDCl}_{3}\right) \delta 210.14,176.22,170.79$, $170.05,62.20,61.36,60.92,58.41,47.76,46.99,42.00,41.95,38.86,18.99,14.42,14.19,14.18$, 14.01. LRMS m/z (\%) 356 (5, [M] $\left.]^{+}\right), 310$ (24), 283 (6), 268 (5), 237 (30), 209 (17), 187 (4), 170 (100), 141 (97), 127 (2), 113 (24), 99 (2), 85 (6), 55 (4). FAB-HRMS: $m / z$ calcd for $\left[\mathrm{C}_{18} \mathrm{H}_{28} \mathrm{O}_{7}+\mathrm{H}\right]^{+}$ 357.1908, found 357.1909. IR $\left(\mathrm{CDCl}_{3}\right): 2963,1727,1186,905,729 \mathrm{~cm}^{-1}$.

cis-2,4-Bis(ethoxycarbonyl)-2-(2-ethoxy-2-oxoethyl)-4-propylcyclopentanone (cis-2b): Colorless oil, $\mathrm{R}_{\mathrm{f}}=0.48\left(15 \%\right.$ EA in PE). ${ }^{1} \mathrm{H}-\mathrm{NMR}\left(\mathrm{CDCl}_{3}\right) \delta 4.21-4.09(\mathrm{~m}, 5 \mathrm{H}), 4.07-4.00(\mathrm{~m}, 1 \mathrm{H}), 3.31$ (dd, $J=19.1$, $1.0 \mathrm{~Hz}, 1 \mathrm{H}), 3.22$ (d, $J=14.2 \mathrm{~Hz}, 1 \mathrm{H}), 2.95$ (d, $J=17.6 \mathrm{~Hz}, 1 \mathrm{H}), 2.89$ (d, $J=17.6 \mathrm{~Hz}, 1 \mathrm{H}), 2.39$ (d, $J=19.1 \mathrm{~Hz}, 1 \mathrm{H}), 2.07(\mathrm{~d}, J=14.2 \mathrm{~Hz}, 1 \mathrm{H}), 1.94-1.84(\mathrm{~m}, 1 \mathrm{H}), 1.64-1.51(\mathrm{~m}, 1 \mathrm{H}), 1.28(\mathrm{t}, J=7.2 \mathrm{~Hz}$, $3 \mathrm{H}), 1.27-1.23(\mathrm{~m}, 5 \mathrm{H}), 1.20(\mathrm{t}, J=7.1 \mathrm{~Hz}, 3 \mathrm{H}), 0.90(\mathrm{t}, J=7.3 \mathrm{~Hz}, 3 \mathrm{H}) .{ }^{13} \mathrm{C}-\mathrm{NMR}\left(\mathrm{CDCl}_{3}\right) \delta 211.79$, $175.65,170.81,169.87,62.08,61.28,61.10,58.08,48.63,46.21,43.94,42.90,39.16,19.17,14.42$, 14.30, 14.25, 13.97. LRMS m/z (\%) 356 (5, [M] $), 310$ (25), 283 (8), 268 (6), 255 (2), 237 (30), 209 (20), 187 (5), 170 (100), 141(99), 127 (3), 113 (27), 99 (3), 85 (6), 71 (2), 55 (5). FAB-HRMS: $m / z$ calcd for $\left[\mathrm{C}_{18} \mathrm{H}_{28} \mathrm{O}_{7}+\mathrm{H}\right]^{+}$357.1908, found 357.1910. IR $\left(\mathrm{CDCl}_{3}\right): 2961,1728,1180,1030,907,730 \mathrm{~cm}^{-1}$.

trans-2,4-Bis(butoxycarbonyl)-2-(2-butoxy-2-oxoethyl)-4-propylcyclopentanone (trans-2c): Colorless oil, $\mathrm{R}_{\mathrm{f}}=0.54\left(10 \%\right.$ EA in PE). ${ }^{1} \mathrm{H}-\mathrm{NMR}\left(\mathrm{CDCl}_{3}\right) \delta 4.17-3.98(\mathrm{~m}, 6 \mathrm{H}), 3.14(\mathrm{~d}, J=17.5 \mathrm{~Hz}, 1 \mathrm{H}), 2.97$ $(\mathrm{d}, J=17.7 \mathrm{~Hz}, 1 \mathrm{H}), 2.86(\mathrm{~d}, J=14.8 \mathrm{~Hz}, 1 \mathrm{H}), 2.65(\mathrm{dd}, J=14.7,0.8 \mathrm{~Hz}, 1 \mathrm{H}), 2.50(\mathrm{~d}, J=17.5 \mathrm{~Hz}$, 1H), $2.43(\mathrm{~d}, J=17.8 \mathrm{~Hz}, 1 \mathrm{H}), 1.90(\mathrm{ddd}, J=13.4,10.5,6.3 \mathrm{~Hz}, 1 \mathrm{H}), 1.70$ (ddd, $J=13.4,10.3,6.5 \mathrm{~Hz}$, $1 \mathrm{H}), 1.64-1.53(\mathrm{~m}, 6 \mathrm{H}), 1.40-1.32(\mathrm{~m}, 6 \mathrm{H}), 1.30-1.25(\mathrm{~m}, 2 \mathrm{H}), 0.99-0.88(\mathrm{~m}, 12 \mathrm{H}) .{ }^{13} \mathrm{C}-\mathrm{NMR}$ $\left(\mathrm{CDCl}_{3}\right) \delta 210.16,176.41,170.95,170.19,66.14,65.33,64.90,58.53,47.95,47.06,42.14,42.11$, $38.90,30.68,30.66,30.55,19.24,19.21,19.17,19.12,14.47,13.81,13.78(2 \mathrm{C})$. LRMS $m / z(\%) 440$ $\left(2,[\mathrm{M}]^{+}\right), 368$ (10), 339 (3), 324 (3), 310 (5), 265 (6), 243 (3), 210 (10), 198 (47), 181 (6), 169 (6), 142 (100), 113 (26), 99 (1), 85 (2), 55 (3). FAB-HRMS: $m / z$ calcd for $\left[\mathrm{C}_{24} \mathrm{H}_{40} \mathrm{O}_{7}+\mathrm{H}\right]^{+}$441.2847, found 441.2847. IR $\left(\mathrm{CH}_{2} \mathrm{Cl}_{2}\right): 2960,1730,1183,909,731 \mathrm{~cm}^{-1}$.

cis-2,4-Bis(butoxycarbonyl)-2-(2-butoxy-2-oxoethyl)-4-propylcyclopentanone (cis-2c): Colorless oil, $\mathrm{R}_{\mathrm{f}}=0.48\left(10 \%\right.$ EA in PE). ${ }^{1} \mathrm{H}-\mathrm{NMR}\left(\mathrm{CDCl}_{3}\right) \delta 4.09(\mathrm{t}, J=6.6 \mathrm{~Hz}, 3 \mathrm{H}), 4.05(\mathrm{t}, J=6.7 \mathrm{~Hz}, 2 \mathrm{H}), 3.97(\mathrm{dt}$, $J=11.1,6.7 \mathrm{~Hz}, 1 \mathrm{H}), 3.30(\mathrm{~d}, J=19.0 \mathrm{~Hz}, 1 \mathrm{H}), 3.22(\mathrm{~d}, J=14.2 \mathrm{~Hz}, 1 \mathrm{H}), 2.96(\mathrm{~d}, J=17.6 \mathrm{~Hz}, 1 \mathrm{H}), 2.88$ $(\mathrm{d}, J=17.6 \mathrm{~Hz}, 1 \mathrm{H}), 2.38(\mathrm{~d}, J=19.0 \mathrm{~Hz}, 1 \mathrm{H}), 2.06(\mathrm{~d}, J=14.2 \mathrm{~Hz}, 1 \mathrm{H}), 1.89$ (td, $J=12.4,4.9 \mathrm{~Hz}$, $1 \mathrm{H}), 1.68-1.51(\mathrm{~m}, 7 \mathrm{H}), 1.43-1.19(\mathrm{~m}, 8 \mathrm{H}), 1.09-0.70(\mathrm{~m}, 12 \mathrm{H}) .{ }^{13} \mathrm{C}-\mathrm{NMR}\left(\mathrm{CDCl}_{3}\right) \delta 211.59,175.65$, 170.87, 169.87, 65.94, 65.22, 64.99, 58.13, 48.70, 46.18, 43.92, 42.95, 39.18, 30.69, 30.66, 30.45, 19.33, 19.19, 19.06, 14.38, 13.83, 13.78. LRMS $m / z(\%) 440$ (2, [M] $]^{+}, 368$ (10), 339 (2), 324 (3), 310 (5), 265 (3), 243 (6), 210 (4), 198 (48), 181 (10), 169 (11), 142 (100), 113 (25), 99 (1), 85 (2), 55 (3). IR $\left(\mathrm{CH}_{2} \mathrm{Cl}_{2}\right): 2959,1758,1176 \mathrm{~cm}^{-1}$.

trans-2,4-Bis(isopropoxycarbonyl)-2-(2-isopropoxy-2-oxoethyl)-4-propylcyclopentanone (trans-2d): Colorless oil, $\mathrm{R}_{\mathrm{f}}=0.44\left(15 \% \mathrm{EA}\right.$ in PE). ${ }^{1} \mathrm{H}-\mathrm{NMR}\left(\mathrm{CDCl}_{3}\right) \delta 5.04-4.92(\mathrm{~m}, 3 \mathrm{H}), 3.09(\mathrm{~d}, J=17.5 \mathrm{~Hz}$, 
1H), $2.95(\mathrm{~d}, J=17.8 \mathrm{~Hz}, 1 \mathrm{H}), 2.83(\mathrm{~d}, J=14.8 \mathrm{~Hz}, 1 \mathrm{H}), 2.63(\mathrm{~d}, J=14.7 \mathrm{~Hz}, 1 \mathrm{H}), 2.46(\mathrm{~d}, J=17.5 \mathrm{~Hz}$, $1 \mathrm{H}), 2.41(\mathrm{~d}, J=17.8 \mathrm{~Hz}, 1 \mathrm{H}), 1.90(\mathrm{ddd}, J=13.3,10.3,6.5 \mathrm{~Hz}, 1 \mathrm{H}), 1.72-1.64(\mathrm{~m}, 1 \mathrm{H}), 1.31-1.19$ $(\mathrm{m}, 20 \mathrm{H}), 0.93(\mathrm{t}, J=7.3 \mathrm{~Hz}, 3 \mathrm{H}) .{ }^{13} \mathrm{C}-\mathrm{NMR}\left(\mathrm{CDCl}_{3}\right) \delta 210.34,175.80,170.34,169.61,69.84,68.81$, 68.44, 58.59, 47.75, 46.96, 42.01, 42.00, 39.11, 21.90, 21.84, 21.75, 21.74, 21.68, 21.52, 19.02, 14.51. LRMS m/z (\%) 398 (1, [M] $\left.]^{+}\right), 355$ (5), 339 (4), 311 (8), 297 (19), 271 (3), 237 (9), 215 (38), 184 (31), 173 (30), 155 (11), 142 (100), 113 (38), 99 (4), 85 (5), 71 (1), 55 (4). FAB-HRMS: $m / z$ calcd for $\left[\mathrm{C}_{21} \mathrm{H}_{34} \mathrm{O}_{7}+\mathrm{H}\right]^{+}:$399.2378, found: 399.2374. IR $\left(\mathrm{CH}_{2} \mathrm{Cl}_{2}\right): 2983,1723,1102,905,726 \mathrm{~cm}^{-1}$.

cis-2,4-Bis(isopropoxycarbonyl)-2-(2-isopropoxy-2-oxoethyl)-4-propylcyclopentanone (cis-2d): Colorless oil, $\mathrm{R}_{\mathrm{f}}=038\left(15 \%\right.$ EA in PE). ${ }^{1} \mathrm{H}-\mathrm{NMR}\left(\mathrm{CDCl}_{3}\right) \delta 5.06-4.89(\mathrm{~m}, 3 \mathrm{H}), 3.28(\mathrm{~d}, J=19.0 \mathrm{~Hz}, 1 \mathrm{H}), 3.21$ $(\mathrm{d}, J=14.4 \mathrm{~Hz}, 1 \mathrm{H}), 2.92$ (d, $J=17.4 \mathrm{~Hz}, 1 \mathrm{H}), 2.81$ (d, $J=17.4 \mathrm{~Hz}, 1 \mathrm{H}), 2.39-2.32(\mathrm{~m}, 1 \mathrm{H}), 2.03(\mathrm{~d}$, $J=14.2 \mathrm{~Hz}, 1 \mathrm{H}), 1.92-1.84(\mathrm{~m}, 1 \mathrm{H}), 1.57-1.49(\mathrm{~m}, 1 \mathrm{H}), 1.32-1.15(\mathrm{~m}, 23 \mathrm{H}) .{ }^{13} \mathrm{C}-\mathrm{NMR}\left(\mathrm{CDCl}_{3}\right) \delta$ 211.80, 175.03, 170.34, 169.29, 69.79, 68.67, 68.61, 58.36, 48.29, 46.01, 43.90, 42.83, 39.53, 21.93, 21.89, 21.86, 21.83, 21.53, 21.51, 19.06, 14.46. LRMS m/z (\%) 398 (2, [M] $\left.]^{+}\right), 355$ (5), 339 (3), 311 (2), 297 (27), 271 (2), 237 (16), 215 (39), 184 (29), 173 (29), 155 (11), 142 (100), 113 (31), 99 (3), 85 (3), 71 (1), 55 (2). FAB-HRMS: $m / z$ calcd for $\left[\mathrm{C}_{21} \mathrm{H}_{34} \mathrm{O}_{7}+\mathrm{H}\right]^{+}$399.2378, found: 399.2378. IR $\left(\mathrm{CH}_{2} \mathrm{Cl}_{2}\right): 2982,1724,1265,1105,735 \mathrm{~cm}^{-1}$.

Reductive Dimerization of 1a

Under a nitrogen atmosphere and stirring, PMHS $(0.12 \mathrm{~mL}, 2.0 \mathrm{mmol} \mathrm{SiH})$ was added to a dried Schlenk tube charged with DPEphos (18.9 mg, $0.035 \mathrm{mmol}),\left[\mathrm{CuF}\left(\mathrm{PPh}_{3}\right)_{3}\right] \cdot 2 \mathrm{MeOH}(28.0 \mathrm{mg}$, $0.030 \mathrm{mmol}), \mathbf{1 a}(0.205 \mathrm{~g}, 1.30 \mathrm{mmol})$ and toluene $(2.0 \mathrm{~mL})$. The mixture was stirred until $\mathbf{1 a}$ was consumption as monitored by TLC. A saturated ammonium fluoride solution in water ( $2 \mathrm{~mL})$ was added to the mixture to quench the reaction. After stirred for $0.5 \mathrm{~h}$, the mixture was separated by phase separation, and the aqueous solution was extracted with dichloromethane $(3 \mathrm{~mL} \times 10 \mathrm{~mL})$. The combined organic phase was dried and analyzed by GC to determine the diastereomeric ratio. Concentration of the dried organic solution and column chromatography gave 3a as a colorless oil ( $0.076 \mathrm{~g}, 41 \%$ yield). Pure diastereomers of 3a could be obtained by further column separation using petroleum ether-ethyl acetate eluent.

trans-2,4-Bis(methoxycarbonyl)-2-(2-methoxy-2-oxoethyl)-4-methylcyclopentanone (trans-3a): $\mathrm{R}_{\mathrm{f}}=0.39$ $\left(20 \%\right.$ EA in PE). ${ }^{1} \mathrm{H}-\mathrm{NMR}\left(\mathrm{CDCl}_{3}\right) \delta 3.61(\mathrm{~s}, 3 \mathrm{H}), 3.59$ (s, 3H), $3.54(\mathrm{~s}, 3 \mathrm{H}), 3.02$ (d, $J=17.5 \mathrm{~Hz}$, 1H), $2.85(\mathrm{~d}, J=17.8 \mathrm{~Hz}, 1 \mathrm{H}), 2.69(\mathrm{~d}, J=14.8 \mathrm{~Hz}, 1 \mathrm{H}), 2.51(\mathrm{~d}, J=14.1 \mathrm{~Hz}, 1 \mathrm{H}), 2.50(\mathrm{~d}, J=17.7 \mathrm{~Hz}$, 1H) $2.35(\mathrm{~d}, J=18.0 \mathrm{~Hz}, 1 \mathrm{H}), 1.37(\mathrm{~s}, 3 \mathrm{H}) .{ }^{13} \mathrm{C}-\mathrm{NMR}\left(\mathrm{CDCl}_{3}\right) \delta 209.48,176.91,170.90,170.25$, 58.46, 52.94, 52.28, 51.67, 48.28, 43.01, 42.91, 38.64, 25.13. MS $m / z(\%): 286\left(3,[\mathrm{M}]^{+}\right), 254(53), 222$ (3), 212 (2), 195 (46), 167 (35), 153 (2), 140 (6), 128 (100), 113 (7), 100 (43), 91 (1), 77 (17), 69 (17), 59 (15), $44(7)$.

cis-2,4-Bis(methoxycarbonyl)-2-(2-methoxy-2-oxoethyl)-4-methylcyclopentanone (cis-3a): $\mathrm{R}_{\mathrm{f}}=0.34$ (20\% EA in PE). ${ }^{1} \mathrm{H}-\mathrm{NMR}\left(\mathrm{CDCl}_{3}\right) \delta 3.72(\mathrm{~s}, 3 \mathrm{H}), 3.67$ (s, 3H), $3.65(\mathrm{~s}, 3 \mathrm{H}), 3.32(\mathrm{~d}, J=18.9 \mathrm{~Hz}, 1 \mathrm{H})$, $3.24(\mathrm{~d}, J=14.2 \mathrm{~Hz}, 1 \mathrm{H}), 2.98(\mathrm{~d}, J=17.6 \mathrm{~Hz}, 1 \mathrm{H}), 2.90(\mathrm{~d}, J=17.6 \mathrm{~Hz}, 1 \mathrm{H}), 2.39$ (d, $J=19.0 \mathrm{~Hz}, 1 \mathrm{H})$, $2.08(\mathrm{~d}, J=14.2 \mathrm{~Hz}, 1 \mathrm{H}), 1.45(\mathrm{~s}, 3 \mathrm{H}) .{ }^{13} \mathrm{C}-\mathrm{NMR}\left(\mathrm{CDCl}_{3}\right) \delta 211.01,176.61,170.95,170.07,58.27$, 
52.78, 52.43, 51.91, 47.75, 44.17, 43.44, 38.73, 26.91. MS m/z (\%): $286\left(4,[\mathrm{M}]^{+}\right), 254$ (50), 222 (3), 212 (2), 195 (45), 167 (26), 153 (1), 140 (6), 128 (100), 113 (6), 100 (23), 91 (1), 79 (13), 69 (15), 59 (11), $41(5)$.

\section{Conclusions}

As a summary, copper-diphosphorous is efficient in catalyzing the diethyl zinc (or a silane)-induced conjugate addition-dimerization/cyclization domino reaction of itaconates, giving 2,4-bis(alkoxycarbonyl)2-(2-alkoxy-2-oxoethyl)-4-alkylcyclopentanones in moderate to high yields. The proportion of dominant diastereomer could be varied by using different copper catalysts.

\section{Supplementary Materials}

Supplementary materials can be accessed at: http://www.mdpi.com/1420-3049/20/08/15023/s1.

\section{Acknowledgments}

We are grateful to NSFC (No. 20972020) and LNET (LR2012041) for financial support.

\section{Author Contributions}

ZNL designed research and wrote the paper. ZQL and RRL performed the experiments. LJ analyzed the spectral data. All authors read and approved the final manuscript.

\section{Conflicts of Interest}

The authors declare no conflict of interest.

\section{References and Notes}

1. Le Notre, J.; van Mele, D.; Frost, C.G. A New Method for Constructing Quaternary Carbon Centres: Tandem Rhodium-Catalysed 1,4-Addition/Intramolecular Cyclisation. Adv. Synth. Catal. 2007, 349, 432-440.

2. Kitamura, M.; Miki, T.; Nakano, K.; Noyori, R. 1,4-Addition of Diorganozincs to $\alpha, \beta$-Unsaturated Ketones Catalyzed by a Copper(I)-Sulfonamide Combined System. Bull. Chem. Soc. Jpn. 2000, 73, 999-1014.

3. Choi, Y.H.; Choi, J.Y.; Yang, H.Y.; Kim, Y.H. Copper-Catalyzed Conjugate Addition on Macrocyclic, Cyclic, and Acyclic Enones with a Chiral Phosphoramidite Ligand Having a $C_{2}$-Symmetric Amine Moiety. Tetrahedron Asymmetry 2002, 13, 801-804.

4. Wilsily, A.; Fillion, E. Asymmetric Synthesis of Carboxylic Acid Derivatives Having an All-Carbon $\alpha$-Quaternary Center through Cu-Catalyzed 1,4-Addition of Dialkylzinc Reagents to 2-Aryl Acetate Derivatives. Org. Lett. 2008, 10, 2801-2804.

5. Ebisu, Y.; Kawamura, K.; Hayashi, M. Enantioselective Copper-Catalyzed 1,4-Addition of Dialkylzincs to Enones Using a Novel N,N,P-Cu(II) Complex. Tetrahedron Asymmetry 2012, 23, 959-964. 
6. Arink, A.M.; Braam, T.W.; Keeris, R.; Jastrzebski, J.T.B.H.; Benhaim, C.; Rosset, S.; Alexakis, A.; van Koten, G. Copper(I) Thiolate Catalysts in Asymmetric Conjugate Addition Reactions. Org. Lett. 2004, 6, 1959-1962.

7. Shan, F.; Jiang, L.; Li, Z.; Zhao, D. Asymmetric Conjugate Addition to Cyclic Enone Catalyzed by Cu-NHC Complexes with $C_{2}$ Symmetry. Chin. J. Chem. 2011, 29, 973-977.

8. Li, J.; You, S.; Cai, M. Synthesis of Tetrasubstituted Olefins by Stereoselective Allylzincation-Negishi Tandem Reaction of Acetylenic Sulfones. J. Chem. Res. 2008, 8, 429-431.

9. Wang, Y.L.; Zhu, S.Z. Reformatsky-Type Aldol Reactions of 4-Bromo-4,4-Difluoroacetoacetate with Aldehydes and Ketones. Tetrahedron Lett. 2001, 42, 5741-5744.

10. Mukaiyama, T.; Kagayama, A.; Igarashi, K.; Shiina, I. Diastereoselective Aldol Reaction of $\alpha$-Bromo Ketones with Aliphatic Aldehydes by Using Titanium(II) Chloride and Copper. Chem. Lett. 1999, $1157-1158$.

11. Du, Y.; Xu, L.W.; Shimizu, Y.; Oisaki, K.; Kanai, M.; Shibasaki, M. Asymmetric Reductive Mannich Reaction to Ketimines Catalyzed by a $\mathrm{Cu}(\mathrm{I})$ Complex. J. Am. Chem. Soc. 2008, 130, 16146-16147.

12. Anderson, J.C.; Blake, A.J.; Koovits, P.J.; Stepney, G.J. Diastereoselective Reductive Nitro-Mannich Reactions. J. Org. Chem. 2012, 77, 4711-4724.

13. Sakanishi, K.; Itoh, S.; Sugiyama, R.; Nishimura, S.; Kakeya, H.; Iwabuchi, Y.; Kanoh, N. Total Synthesis of the Proposed Structure of Heronamide C. Eur. J. Org. Chem. 2014, 2014, 1376-1380.

14. Bausch, C.C.; Johnson, J.S. Conjugate Addition/Ireland-Claisen Rearrangements of Allyl Fumarates: Simple Access to Terminally Differentiated Succinates. J. Org. Chem. 2008, 73, 1575-1577.

15. Li, Z.; Zhang, Z.; Yuan, L.; Jiang, L.; Li, Z.; Li, Z. Copper Hydride-Catalyzed Reductive Aldol Addition-Lactonization Domino Reactions of $\alpha, \beta$-Unsaturated Diesters. Synlett 2014, 25, 724-728.

16. Li, Z.; Feng, Y.; Li, Z.; Jiang, L. Copper-Catalyzed Three-Component Tandem Reactions for the Synthesis of $\beta$-Carboalkoxy- $\gamma$-Lactams. Synlett 2014, 25, 2899-2902.

17. Yamaguchi, K.; Yokota, K.; Takada, Y. Cyclodimerization of Dimethyl Methylenesuccinate by Alkylaluminum Compounds. Chem. Lett. 1984, 13, 543-546.

18. Tolstikov, G.A.; Miftakhov, M.S.; Akbutina, F.A. Key Lactone for 11-Methylprostaglandins. Zh. Org. Khim. 1985, 21, 674-675.

19. Johnson, F.; Paul, K.G.; Favara, D.; Ciabatti, R.; Guzzi, U. Prostaglandins. 2. Synthesis of Prostaglandin F2. $\alpha$. In Optically Active Form from Chiral Precursors. J. Am. Chem. Soc. 1982, 104, 2190-2198.

20. Magriotis, P.A.; Johnson, F. Steroids. 3. A New Synthetic Approach to Optically Active Steroids. Total Synthesis of (+)-18-Hydroxyestrone. J. Org. Chem. 1984, 49, 1460-1461.

21. Node, M.; Nakamura, D.; Nishide, K.; Inoue, T. A Facile Asymmetric Synthesis of Corey Lactone Utilizing $C_{2}$-Symmetric Dimethyl 3,7-Dihydroxy-cis-bicyclo[3.3.0]octan-2,6-diene-2,6-dicarboxylate. Heterocycles 1997, 46, 535-540.

22. Mitani, M.; Pakjamsai, C.; Tsuchida, T.; Kudoh, H. Oligomerization of Acrylic Acid Derivatives by a Reaction with the Alkylcopper(I)-Phosphine Complex. J. Chem. Res. 2000, 4-5, doi:10.3184/030823400103165635.

23. Cis- and trans- refer to the relationships of the two carboxylates linked to the cyclopentanone. 
24. The structure of the less polar product is assigned as trans-2a, in which the protons of $\mathrm{C}_{2} \mathrm{CO}_{2} \mathrm{CH}_{3}(\delta=3.15,2.54 \mathrm{ppm})$ have weaker or no correlations with the $\mathrm{C}_{2} \mathrm{CH}_{2} \mathrm{CH}_{3}(\delta=1.88$, $1.71 \mathrm{ppm}$ ) in the NOESY spectrum. For the more polar product, the protons of $\mathrm{CH}_{2} \mathrm{CO}_{2} \mathrm{CH}_{3}$ $(\delta=2.96,2.91 \mathrm{ppm})$ have correlations with the signals of $\mathrm{C}_{2} \mathrm{CH}_{2} \mathrm{CH}_{3}(\delta=1.92,1.56 \mathrm{ppm})$. Therefore, There is a cis-realationship between $\mathrm{CH}_{2} \mathrm{CH}_{2} \mathrm{CH}_{3}$ and $\mathrm{CH}_{2} \mathrm{CO}_{2} \mathrm{CH}_{3}$, and the structure of the more polar product is deduced as cis-2a.

25. Mori, A.; Fujita, A.; Nishihara, Y.; Hiyama, T. Copper(I) Salt Mediated 1,4-Reduction of $\alpha, \beta$-Unsaturated Ketones Using Hydrosilanes. Chem. Commun. 1997, 2159-2160.

26. Li, Z.; Jiang, L.; Li, Z.; Chen, H. Copper Hydride-Catalyzed Conjugate Reduction-Aldol Addition Domino Reaction of $\alpha, \beta-$ Unsaturated Carboxylates with Ketones. Chin. J. Chem. 2013, 31, 539-544.

27. Zheng, A.J.; Shan, F.J.; Li, Z.N.; Li, Z.C.; Jiang, L. Copper Hydride-Catalyzed Reduction of Electron-Deficient Olefins. Chem. Pap. 2013, 67, 1271-1276.

28. Yamaguchi, K.; Yokota, K. Oligomerization of Allyl Methacrylate in the Presence of Ethylaluminum Compounds and Isolation of Its Unimer and Dimer. Polym. J. 1993, 25, 639-643.

29. Brown, M.K.; Degrado, S.J.; Hoveyda, A.H. Highly Enantioselective Cu-Catalyzed Conjugate Additions of Dialkylzinc Reagents to Unsaturated Furanones and Pyranones: Preparation of Air-Stable and Catalytically Active Cu-Peptide Complexes. Angew. Chem. Int. Ed. 2005, 44, 5306-5310.

30. Baker, B.A.; Bokovic, Z.V.; Lipshutz, B.H. (BDP)CuH: A "Hot" Stryker's Reagent for Use in Achiral Conjugate Reductions. Org. Lett. 2008, 10, 289-292.

31. Li, N.; Ou, J.; Miesch, M.; Chiu, P. Conjugate Reduction and Reductive Aldol Cyclization of $\alpha, \beta-$-Unsaturated Thioesters Catalyzed by (BDP)CuH. Org. Biomol. Chem. 2011, 9, 6143-6147.

Sample Availability: Samples of the compounds $\mathbf{2 a - 2 d}$ are available from the authors.

(C) 2015 by the authors; licensee MDPI, Basel, Switzerland. This article is an open access article distributed under the terms and conditions of the Creative Commons Attribution license (http://creativecommons.org/licenses/by/4.0/). 